\title{
Nuclear modification of full jet energy and jet structure at the LHC energies
}

\author{
Ning-Bo Chang, ${ }^{a, *}$ Guang-You Qin ${ }^{b}$ and Yasuki Tachibana ${ }^{c}$ \\ ${ }^{a}$ Institute of Theoretical Physics, Xinyang Normal University, Xinyang, Henan 464000, China \\ ${ }^{b}$ Institute of Particle Physics, Central China Normal University, Wuhan 430079, China \\ ${ }^{c}$ Akita International University, Akita, 010-1292 Japan \\ E-mail: changnb@xynu.edu.cn, guangyou.qin@mail.ccnu.edu.cn, \\ ytachibana@aiu.ac.jp
}

With our coupled jet-fluid model, we study the evolution of the full jet shower in quark-gluon plasma (QGP) and calculate the observables related to the nuclear modification of jet energy and jet structure in $\mathrm{Pb}+\mathrm{Pb}$ collisions at 2.76 $\mathrm{ATeV}$ and 5.02 ATeV. The full jet shower evolution in QGP medium is described by a set of coupled Boltzmann transport equations which includes the effects of collisional energy loss, transverse momentum broadening and medium-induced splitting process. The jet observed in heavy-ion collisions also includes the particles from the QGP medium excitated by the energy and momentum transported from jet shower to QGP medium. To take account of this effect, the dynamical evolution of QGP medium need to be simulated by solving relativistic hydrodynamic equation with source terms which is provided by the jet evolution equations. Our results can describe the experimental data of jet nuclear modification factor $R_{A A}$ with different cone size and catch the features of jet shape modification for inclusive jets and $\gamma$-jets. Our study demonstrates that the effect of medium response is essential for the cone size dependence of jet energy loss and jet $R_{A A}$, and becomes important for the modification of jet shape function at large radius. For the different modification pattern of the jet shape function in single inclusive jet events and $\gamma$-jet events observed by the CMS Collaboration, our results show that the difference comes from the dependence on jet energy instead of the flavor of the parton that initiates the jet. Our theory can be tested in the future by measuring the modification of jet shape function over a wider range of jet energy in heavy-ion collisions.

HardProbes 2020

1-6 June 2020

Austin, Texas

*Speaker 


\section{Introduction}

Jet quenching is the main hard probe to study the properties of the quark gluon plasma (QGP) created in ultra-relativistic heavy-ion collisions. In earlier studies people mainly focus on the quenching of leading particles in jets, but since ten years ago full jets can be reconstructed from the large fluctuating background, the study on the quenching of full jets has became the center of this area. The reason is that full jets can provide us more observables and tell us more information about the jet medium interactions than leading particles. The observables from leading particles like the suppression of hadrons spectra can only reflect the energy loss of the leading partons in jets. While the observables of full jets can not only reflect the energy loss of the full jets, like the suppression of jet spectra, and also reflect the energy redistribution in jets, like the modification of jet fragmentation function and jet shape function.

When a partonic jet shower passes through the QGP medium all partons in the shower suffer elastic and inelastic scatterings with the medium partons. Our coupled jet-fluid model [1-3] takes into account the effects of collisional energy loss and transverse momentum broadening caused by elastic scatterings and the process of medium induced radiation due to inelastic scatterings. And in the process of jet propagation, the energy transported from jets to medium evolves with the medium hydrodynamically, may feed back to jets in the process of jet reconstruction. This effect is called medium response, which is also included in our model because it may be important to the jet energy distribution at large radius. The CMS collaboration measures the modification of jet shape function in $\mathrm{Pb}+\mathrm{Pb}$ collisions for single inclusive jets $[4,5]$ and for $\gamma$-jets [6], and the measurements show different modification pattern which has attracted lots of attention. In this proceeding, we will discuss this problem after presenting our framework and the results about jet energy loss.

\section{Framework}

In our coupled jet-fluid model [1-3], we simulate the jet shower evolution via solving the coupled Boltzmann transport equations for $f_{i}\left(\omega_{i}, k_{i \perp}^{2}\right)=d N_{i}\left(\omega_{i}, k_{i \perp}^{2}\right) / d \omega_{i} d k_{i \perp}^{2}$, which is the threedimensional momentum distribution of quarks and gluons in jets, with $\omega_{i}$ the energy of parton $i$ and $k_{i \perp}$ its transverse momentum with respect to the jet axis. With $f_{i}\left(\omega_{i}, k_{i \perp}^{2}\right)$ we can construct many observables, such as jet energy and jet shape function in a defined cone size. The Boltzmann transport equation reads,

$$
\begin{aligned}
\frac{d}{d t} f_{i}\left(\omega_{i}, k_{i \perp}^{2}, t\right)= & \left(\hat{e}_{i} \frac{\partial}{\partial \omega_{i}}+\frac{1}{4} \hat{q}_{i} \nabla_{k_{\perp}}^{2}\right) f_{i}\left(\omega_{i}, k_{i \perp}^{2}, t\right) \\
& +\sum_{j} \int d \omega_{j} d k_{j \perp}^{2} \frac{d \tilde{\Gamma}_{j \rightarrow i}\left(\omega_{i}, k_{i \perp}^{2} \mid \omega_{j}, k_{j \perp}^{2}\right)}{d \omega_{i} d^{2} k_{i \perp} d t} f_{j}\left(\omega_{j}, k_{j \perp}^{2}, t\right) \\
& -\sum_{j} \int d \omega_{j} d k_{j \perp}^{2} \frac{d \tilde{\Gamma}_{i \rightarrow j}\left(\omega_{j}, k_{j \perp}^{2} \mid \omega_{i}, k_{i \perp}^{2}\right)}{d \omega_{j} d^{2} k_{j \perp} d t} f_{i}\left(\omega_{i}, k_{i \perp}^{2}, t\right),
\end{aligned}
$$

where the first and second terms represent the effects of collisional energy loss and transverse momentum broadening due to elastic scatterings, the last two terms represent the generation and disappearance of parton $i$ due to medium induced radiations with $\frac{d \tilde{\Gamma}_{j \rightarrow i}}{d \omega d^{2} k_{\perp} d t}$ the splitting kernel taken 
from the higher twist formalism [7]. In this study all splitting kernels are included, so quark and gluon can convert into each other in the evolution, and the differential equations for quark and gluon distribution must be solved simultaneously.

To solve the evolution equations, the initial parton distribution $f_{i}\left(\omega_{i}, k_{i \perp}^{2}\right)$ must be provided. We generate it using PYTHIA and tune the parameters to make sure the experimental data of jet shape function in $\mathrm{p}+\mathrm{p}$ collisions can be described. There are two parameters in the evolution equation, $\hat{e}$ and $\hat{q}$, which are assumed to have the relation $\hat{q}=4 T \hat{e}$ for simplicity. $\hat{q}$ can be calculated from the local temperature and flow velocity of the QGP medium, $\hat{q}(\tau, \vec{r})=\hat{q}_{0} \cdot \frac{T^{3}(\tau, \vec{r})}{T_{0}^{3}\left(\tau_{0}, \overrightarrow{0}\right)} \cdot \frac{p \cdot u(\tau, \vec{r})}{p_{0}}$, with $\hat{q}_{0}$ the parameter to be fixed by experimental data.

In the process of jet evolution, jets exchange energy and momentum with the QGP medium via elastic scatterings, which also modifies the evolution of the QGP medium and in turn affects the jet reconstruction process because the background is modified. To calculate the contribution of medium response to jets, we firstly solve the the hydrodynamic equation with source term [2], $\partial_{\mu} T_{\text {fluid }}^{\mu v}(x)=J^{v}(x)$, with

$$
J^{v}(x)=\sum_{i} \int \frac{d \omega_{i} d k_{i \perp}^{2} d \phi_{i}}{2 \pi} \delta^{(3)}\left(\mathbf{x}-\mathbf{x}_{0}^{\mathrm{jet}}-\frac{\mathbf{k}_{i}}{\omega_{i}} t\right) \times k_{i}^{v}\left(\hat{e}_{i} \frac{\partial}{\partial \omega_{i}}+\frac{1}{4} \hat{q}_{i} \nabla_{k_{\perp}}^{2}\right) f_{i}\left(\omega_{i}, k_{i \perp}^{2}, t\right),
$$

calculated using the first two terms in Eq. (1). Then we solve the hydrodynamic equation without source term, and calculate the difference of the hadron spectra from the hadronization of the QGP medium with and without the source term. From the hadron spectra induced by the source term, we can calculate their contribution to jet energy and jet shape function.

\section{Results}

At first step, we calculate the nuclear modification factor of jet spectra, i.e., jet $R_{A A}$ to fix the value of $\hat{q}_{0}$. With the experimental data of jets with cone size $R=0.3$, we obtain that the value of $\hat{q}_{0}$ in central $\mathrm{Pb}+\mathrm{Pb}$ collisions is $1.7 \mathrm{GeV}^{2} / \mathrm{fm}$ at $2.76 \mathrm{ATeV}$ and $1.8 \mathrm{GeV}^{2} / \mathrm{fm}$ at $5.02 \mathrm{ATeV}[2,3]$. Then we calculate the jet $R_{A A}$ with different cone size. As shown in Fig. 1 we calculate the ratio of jet $R_{A A}$ with cone size $0.3-0.5$ to that of 0.2 at $2.76 \mathrm{ATeV}$, and calculate the jet $R_{A A}$ with cone size 0.2-0.4 at 5.02 ATeV. From Fig. 1 one can find that only when the effect of medium response is included, there's cone size dependence in the theoretical results. At $2.76 \mathrm{ATeV}$ our theoretical results can describe the ATLAS data [8] better. At $5.02 \mathrm{ATeV}$ though the results with medium response are closer to the center points of the ALICE data [9], the error bar of the data is too large to draw a conclusion.

Jet $R_{A A}$ reflects the total energy loss of full jets, and its dependence on cone size implies that medium response is important at large radius. The modification of jet shape, on the other hand, can tell us how energy is redistributed in radial direction and show the effect of medium response more directly. Fig. 2 shows the comparison of our results with the experimental data from CMS [4-6]. From Fig. 2 we can see that the modification in inclusive jet events and $\gamma$-jet events show different modification pattern. For inclusive jets there is a dramatic dip at middle radius $r$ while for $\gamma$-jets the modification factor increases almost monotonically as $r$. Our results can catch the features of the measurements and one can find that the effect of medium response becomes important at large $r$. But why our results can describe two different modification pattern simultaneously? 

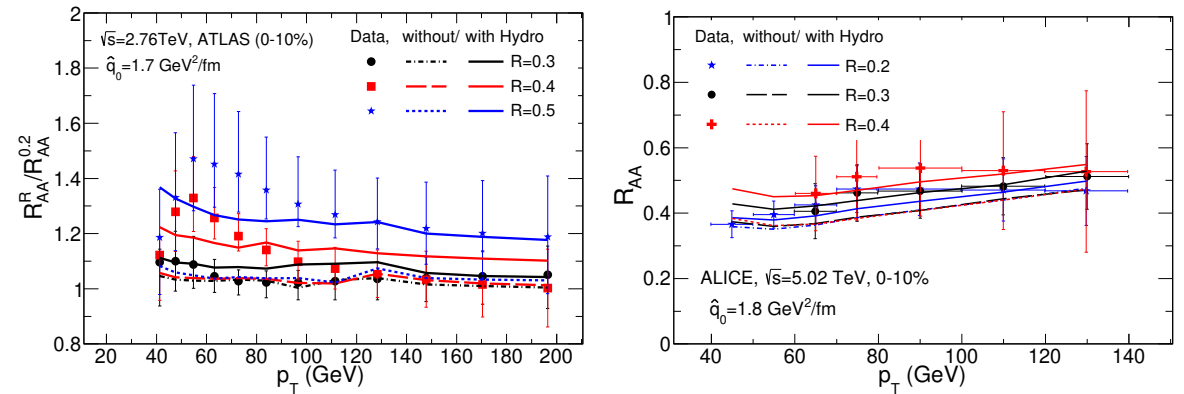

Figure 1: (Left) Ratio of $R_{A A}$ at $2.76 \mathrm{ATeV}$. (Right) $R_{A A}$ with different cone size at $5.02 \mathrm{ATeV}$. Note that 'with/without hydro.' means with or without the effect of medium response. Experiment data comes from Refs. [8, 9].
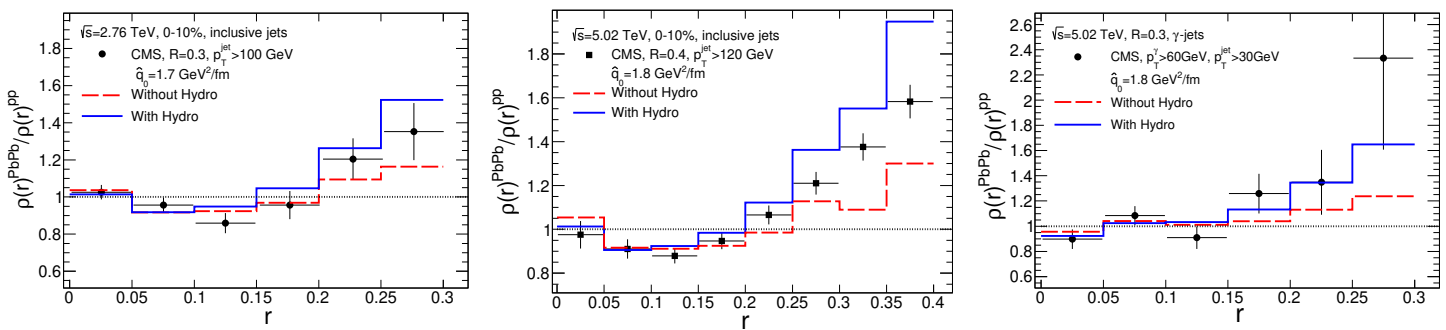

Figure 2: Modification of jet shape for inclusive jets at $2.76 \mathrm{ATeV}$ (left) and $5.02 \mathrm{ATeV}$ (middle), and that for $\gamma$-jets at $5.02 \mathrm{ATeV}$ (right). Experiment data comes from Refs.[4-6].

There are two main differences between the inclusive jets and $\gamma$-jets shown in Fig. 2, the flavor constituents and the range of jet transverse momentum $\left(p_{T}\right)$. To test which factor determines the modification pattern, we calculate the modification of jet shape with medium response for $\gamma$-jets and inclusive jets with two $p_{T}$ cut, $30 \mathrm{GeV}$ and $100 \mathrm{GeV}$, at two collision energies, as shown in Fig. 3 . One can see that in all cases, the modification factor for jets with $p_{T}>30 \mathrm{GeV}$ is a monotonic function of $r$ and for jets with $p_{T}>100 \mathrm{GeV}$ an non-monotonic function, no matter the jet flavor and collision energy. The reason is that as jet energy decreases the jet core becomes softer and earlier to be modified $[1,3]$.

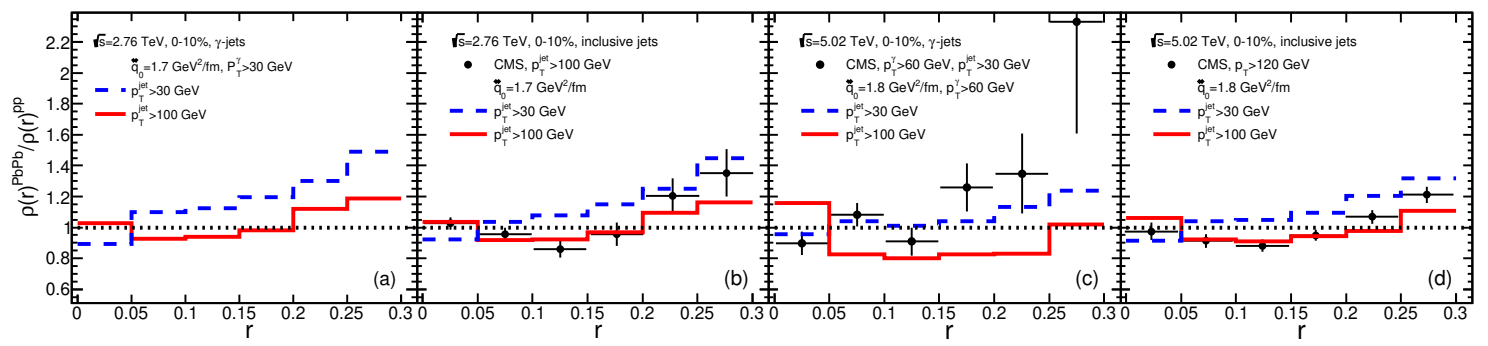

Figure 3: Modification of jet shape for $\gamma$-jets or inclusive jets with jet $p_{T}$ cut $30 \mathrm{GeV}$ and $100 \mathrm{GeV}$, at $2.76 \mathrm{ATeV}$ and $5.02 \mathrm{ATeV}$. 


\section{Summary}

In this proceeding, we present the results of full jet modifications in $\mathrm{Pb}+\mathrm{Pb}$ collisions at 2.76 $\mathrm{ATeV}$ and 5.02 ATeV using our coupled jet-fluid model. In this model the effect of collisional energy loss, transverse momentum broadening and medium induced radiation are included by the coupled Boltzmann transport equations, and the energy and momentum transported from jets to QGP medium is considered as the source term of hydrodynamic evolution to calculate the effect of medium response. Our numerical results can describe jet $R_{A A}$ with different cone size well. The effect of medium respond is important to cone size dependence of jet $R_{A A}$ and jet shape modification at large $r$. For the different modification pattern of jet shape observed by CMS for inclusive jets and $\gamma$-jets, we find that actually the dependence on jet energy determines the modification pattern. Further measurements with wider energy range can test our theory.

Acknowledgements: This work is supported by the Natural Science Foundation of China (NSFC) under Grant Nos. 1775095 and 11905180.

\section{References}

[1] N.-B. Chang and G.-Y. Qin, Full jet evolution in quark-gluon plasma and nuclear modification of jet production and jet shape in Pb+Pb collisions at 2.76ATeV at the CERN Large Hadron Collider, Phys. Rev. C94 (2016) 024902 [1603.01920].

[2] Y. Tachibana, N.-B. Chang and G.-Y. Qin, Full jet in quark-gluon plasma with hydrodynamic medium response, Phys. Rev. C95 (2017) 044909 [1701.07951].

[3] N.-B. Chang, Y. Tachibana and G.-Y. Qin, Nuclear modification of jet shape for inclusive jets and $\gamma$-jets at the LHC energies, Phys. Lett. B 801 (2020) 135181 [1906.09562].

[4] CMS Collaboration collaboration, Modification of jet shapes in PbPb collisions at $\sqrt{s_{N N}}=$ 2.76 TeV, Phys.Lett. B730 (2014) 243 [1310 . 0878].

[5] CMS collaboration, Jet properties in $\mathrm{PbPb}$ and pp collisions at $\sqrt{s_{\mathrm{NN}}}=5.02 \mathrm{TeV}$, JHEP 05 (2018) 006 [1803.00042].

[6] CMS collaboration, Jet Shapes of Isolated Photon-Tagged Jets in Pb-Pb and pp Collisions at $\sqrt{s_{\mathrm{NN}}}=5.02 \mathrm{TeV}$, Phys. Rev. Lett. 122 (2019) 152001 [1809.08602].

[7] X.-N. Wang and X.-F. Guo, Multiple parton scattering in nuclei: Parton energy loss, Nucl. Phys. A696 (2001) 788 [hep-ph/0102230].

[8] ATLAS collaboration, Measurement of the jet radius and transverse momentum dependence of inclusive jet suppression in lead-lead collisions at $\sqrt{s_{N N}}=2.76 \mathrm{TeV}$ with the ATLAS detector, Phys. Lett. B719 (2013) 220 [1208 . 1967].

[9] ALICE collaboration, Measurements of inclusive jet spectra in pp and central $\mathrm{Pb}$-Pb collisions at $\sqrt{s_{\mathrm{NN}}}=5.02 \mathrm{TeV}$, Phys. Rev. C 101 (2020) 034911 [1909.09718]. 